\title{
Implementation and Analysis of Multi-user MIMO with Amplify-and-Forward Relaying
}

\author{
Qiongjie Lin, Yong Jun Chang, Feng Wang and Mary Ann Weitnauer \\ School of Electrical and Computer Engineering \\ Georgia Institute of Technology, Atlanta, Georgia 30332-0250 \\ Email: lin3@gatech.edu; yongjun.chang@gatech.edu; fwang73@gatech.edu; maweit@gatech.edu
}

\begin{abstract}
A two-hop multi-user multiple-inputmultiple-output (MU-MIMO) system with multiple single-antenna amplify-and-forward (AF) relays is demonstrated for the first time in a realistic indoor environment with a commercial software radio platform, using OFDM. Good trend correspondence is observed between the averaged error rate results of experiment and simulation, when zero forcing (ZF) detection is employed and a simple constraint on the average power per relay is used. The challenges of relaying a regenerated digital header and simply amplified spatially multiplexed payload are discussed. It is proven theoretically that the effect of the growth of the number of fixed-gain relays is to bring the destination into the location of the relays. For the case of variable gain, subject to a per-node average power constraint, the destination is also brought asymptotically closer, but not as close as the fixed-gain. However, the gap is approximately closed by allowing relays to deactivate if their gain exceeds a threshold.
\end{abstract}

Index Terms-MU-MIMO, AF, Software-defined Radios (SDRs).

\section{INTRODUCTION}

Multi-user multiple-input-multiple-output (MUMIMO) is a popular and spectrally efficient approach in current cellular standards (e.g., LTE and 802.16m), wherein a multi-antenna base station (BS) serves several users simultaneously in the same wireless channel [1]. MU-MIMO can also relieve congestion at the Sink node in a wireless sensor network [2]. Many authors have considered improving MU-MIMO through the use of either a single multi-antenna amplify-and-forward (AF) relay [3], [4] or multiple single-antenna AF relays [5], [6]. AF relays can potentially reduce latency at both the physical and link layers on the uplink. At the physical layer, they should require less processing time, because they do not need to demultiplex and decode the data. When AF relaying is used to extend range, by utilizing a large number of relays and base station antennas, latency reduction should be possible at Layer 2 of packet networks because of fewer collisions. The two-hop AF network behaves like a pipe: a large number of users can be scheduled to transmit their data simultaneously in the first hop and their packets will not require contention resolution in the second hop. Also, if user cooperation is possible, AF relaying would enable cooperators to be recruited as needed, even when a cooperators number of antennas is less than the number of streams being relayed.

Most of the papers listed above are concerned with optimization of complex relay gains, precoding matrices, or power allocation among sources and relays, and [3], [4], [5] assume globally known channel state information (CSI). The authors in [6] assume a constant gain in each of $L$ AF relay clusters such that a sum-transmit power constraint is satisfied by each whole relay cluster, almost surely. They consider how capacity asymptotically scales with the transmit power and number of hops, as the number of users, relays, and BS antennas also approach infinity. In contrast, our paper treats only $L=1$, and considers how the SNR advantage grows as the number of relays grows, but the number of users and BS antennas is held constant. The authors of [7] consider practical issues, such as preamble design. Apparently, all of these papers evaluate designs through either analysis or computer simulation. In contrast, this paper reports what is apparently the first evaluation of MU-MIMO with AF relaying, on a testbed consisting of software defined radios (SDRs).

We implement a simple variable relay gain based on an average relay transmit power constraint. We show the error rate for two OFDM users, with up to six singleantenna AF relays, and a two-element MIMO receiver at the destination, assuming no direct connection exists between the users and the MIMO destination. The paper presents a novel design of AF transceiver; in particular 
the paper addresses the synchronization challenge in the AF transceiver of separating and later rejoining the header, which is digitally regenerated, with the payload, which is not regenerated. The paper also shows the novel theoretical result that as the number of co-located fixed-gain relays grows, the effect is to bring the users up to the location of the relays. We also consider, via simulation, a simple threshold test on node variable gains to prevent certain relays from inserting excessive noise; this approach appears to bring the variable and fixed gain results very close as the number of relays grows.

\section{SYSTEM MODEL}

We assume the channel is Rayleigh frequency-flat, and slow fading. The zero mean complex Gaussian channel gain $h_{i, k}$ between two nodes $i$ and $k$ satisfies $E\left[\left|h_{i, k}\right|^{2}\right]=\sigma_{0}^{2} d_{i, k}^{-\beta}$, where $\sigma_{0}$ is constant, $\beta$ is the path loss exponent (we use $\beta=3$ ), and $d_{i, k}$ is the distance between nodes $i$ and $k$. As illustrated in Fig. 1, the MUMIMO system consists of a source cluster with M singleantenna users, a relay cluster with a set of single-antenna relay nodes $\left\{R_{1}, \ldots, R_{K}\right\}$, and a $\mathrm{BS}$ with $\mathrm{N}$ antennas, such that $M \leq N$. The users are assumed to be colocated and so are relays, yet all nodes are separated enough to have independent fading. In our case, the relay operates in AF mode in which the source's payload is amplified without any sort of signal regeneration before forwarding to the destination.
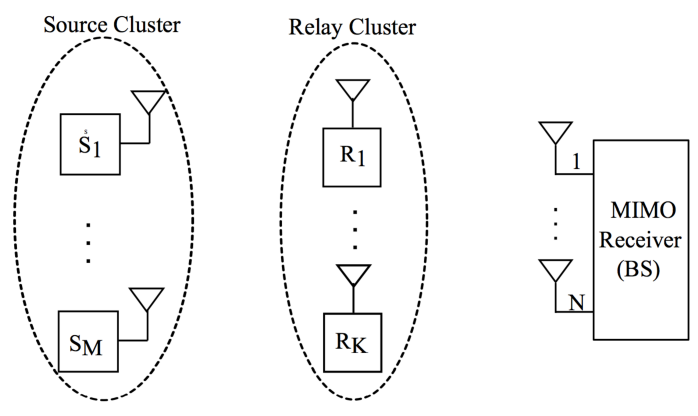

Figure 1: MU-MIMO with AF relays

The multiple symbols of data $\mathbf{x}_{\mathbf{s}}=\left[x_{1}, x_{2}, \ldots, x_{M}\right]^{T}$ from different users in the source cluster go through the $\mathrm{AF}$ relay cluster, which produces at the BS:

$$
y_{D}=H_{2} G H_{1} x_{s}+H_{2} G n_{R}+n_{D},
$$

which can be re-written in terms of the compound channel matrix $\mathcal{H}$ between the source cluster and BS, and the compound noise vector $\boldsymbol{n}_{\boldsymbol{c}}$ as,

$$
\boldsymbol{y}_{D}=\mathcal{H} \boldsymbol{x}_{s}+\boldsymbol{n}_{c}
$$

where

$$
\begin{gathered}
\mathcal{H}=H_{2} G H_{1} \\
n_{c}=H_{2} G n_{R}+n_{D} .
\end{gathered}
$$

$\boldsymbol{H}_{1}$ is the $K \times M$ channel matrix between the source cluster and relay cluster, and $\boldsymbol{H}_{\mathbf{2}}$ represents the $N \times K$ channel matrix between the relay cluster and the BS. $\boldsymbol{n}_{\boldsymbol{R}}$ and $\boldsymbol{n}_{\boldsymbol{D}}$ denote the additive white Gaussian noise (AWGN) at the relays and BS, respectively. The $K \times K$ diagonal matrix $G$ contains the variable amplify gains for the AF relays, such that $g_{k}=\boldsymbol{G}(\boldsymbol{k}, \boldsymbol{k})$, and

$$
g_{k}=\sqrt{\frac{P_{k}}{\left(\sum_{i=1}^{L}\left|y_{k, i}^{\prime}\right|^{2}\right) / L}},
$$

where $P_{k}$ is the transmit power for relay $R_{k}$, and $y_{k, i}^{\prime}$ is the $i t h$ out of L time domain samples of payload received by Relay $k$. We let all relay nodes have same average transmit power as source nodes, that is, $P_{k}=P_{0}$ with $\mathrm{k}=1, \ldots, K$. Here, $P_{0}$ is the transmit power of each user in the source cluster.

\section{Analysis And Simulation Results}

We analyze the BER performance of zero forcing (ZF) detection with binary phase shift keying (BPSK) modulation for the MU-MIMO with AF relays by Monte Carlo simulation. We prove that there is a limit for the performance when the number of relays grows.

We assume the compound channel $\mathcal{H}$ and the covariance matrix $\mathbf{R}=E\left[\boldsymbol{n}_{\boldsymbol{c}} \boldsymbol{n}_{\boldsymbol{c}}^{H}\right]$ of the compound noise $\boldsymbol{n}_{\boldsymbol{c}}$ are known. Based on the Cholesky factorization,

$$
\mathbf{R}=\mathbf{R}^{\mathbf{1 / 2}} \mathbf{R}^{* / 2},
$$

where $\mathbf{R}^{\mathbf{1 / 2}}$ is the lower triangular factor and $\mathbf{R}^{* / 2}$ is the upper trianglular factor, and $\mathbf{R}^{-/ 2}=\left[\mathbf{R}^{\mathbf{1 / 2}}\right]^{-1}$, we have

$$
R^{-/ 2} y_{D}=\underbrace{R^{-/ 2} \mathcal{H}}_{\tilde{H}} x_{s}+\underbrace{R^{-/ 2} n_{c}}_{\tilde{n}},
$$

where $\tilde{\mathbf{H}}$ is the whitened channel and $\tilde{\mathbf{n}}$ is white noise. For this analysis, we suppose the fixed gain scheme [8] for the relays, that is, $\mathbf{G}=g_{0} \mathbf{I}$, where $g_{0}$ is a real-valued scalar.

The instantaneous signal to noise ratio (SNR) for the first user is

$$
\begin{aligned}
\gamma_{1} & =\frac{E_{s}}{N_{0}} \frac{1}{\left\{\left[\tilde{\boldsymbol{H}}^{H} \tilde{\boldsymbol{H}}\right]^{-1}\right\}_{11}} \\
& =\frac{E_{s}}{N_{0}} \frac{1}{\left\{\left[\boldsymbol{H}_{\mathbf{1}}^{H} g_{0} \boldsymbol{H}_{\mathbf{2}}^{H}\left(g_{0}^{2} \boldsymbol{H}_{\mathbf{2}} \boldsymbol{H}_{2}^{H}+\boldsymbol{I}\right)^{-1} \boldsymbol{H}_{\mathbf{2}} g_{0} \boldsymbol{H}_{\mathbf{1}}\right]^{-1}\right\}_{11}},
\end{aligned}
$$


where $E_{s}$ is the transmit energy per symbol period by user node and $N_{0}$ is the power density of AWGN at relay nodes and BS. The development for the second user is same as the first user. For BPSK, the BER is $P_{e}=Q\left(\sqrt{2 \gamma_{1}}\right)$ [9], where $Q(\cdot)$ is the tail probability of the standard normal distribution.

\section{A. Asymptotic SNR as number of relays increases}

If we fix the number of users to be $M$ and antennas of BS to be $N$, and increase the number of the relays, $K$, we find that there is a limit for the $\mathrm{ZF}$ detection performance when $K$ goes to infinity.

Let $\mathbf{M}=g_{0} \mathbf{H}_{2}^{H}\left(g_{0}^{2} \mathbf{H}_{2} \mathbf{H}_{2}^{H}+\mathbf{I}\right)^{-1} \mathbf{H}_{2} g_{0}$, and let the eigenvalue decomposition be $\mathbf{M}=\mathbf{U} \boldsymbol{\Sigma} \mathbf{U}^{\mathbf{H}}$. Then

$$
\begin{aligned}
\gamma_{1} & =\frac{E_{s}}{N_{0}} \frac{1}{\left\{\left[\boldsymbol{H}_{1}^{H} \boldsymbol{M} \boldsymbol{H}_{1}\right]^{-1}\right\}_{11}} \\
& \cong \frac{E_{s}}{N_{0}} \frac{1}{\left\{\left[\boldsymbol{H}_{1}^{H} \boldsymbol{\Sigma} \boldsymbol{H}_{1}\right]^{-1}\right\}_{11}},
\end{aligned}
$$

where $\cong$ means "equivalent in statistics".

For $K>N, \mathbf{M}$ has rank $N$ and each off-diagonal element of $\mathbf{H}_{2} \mathbf{H}_{\mathbf{2}}^{\mathbf{H}}$ is $K$ times a sample cross-correlation of two independent, zero mean white sequences. As $K \rightarrow \infty$, the I matrix becomes insignificant and it follows that $\boldsymbol{\Sigma} \rightarrow\left[\begin{array}{rr}\mathbf{I}_{N} & 0 \\ 0 & 0\end{array}\right]$ and

$$
\begin{aligned}
\lim _{K \rightarrow \infty} \gamma_{1} & =\lim _{K \rightarrow \infty} \frac{E_{s}}{N_{0}} \frac{1}{\left\{\left[\boldsymbol{H}_{1}^{H} \boldsymbol{M} \boldsymbol{H}_{1}\right]^{-1}\right\}_{11}} \\
& =\frac{E_{s}}{N_{0}} \frac{1}{\left\{\left[\overline{\boldsymbol{H}}^{H} \overline{\boldsymbol{H}}\right]^{-1}\right\}_{11}},
\end{aligned}
$$

where $\overline{\mathbf{H}}$ is an $N \times M$ matrix, such that $\overline{\mathbf{H}}^{\mathbf{H}} \overline{\mathbf{H}}$ has the same distribution as $\mathbf{H}_{\mathbf{1}}^{\mathbf{H}} \mathbf{H}_{\mathbf{1}}$. Thus the effect of infinitely many relays is to bring the destination closer to the source to the location of the relay cluster. The performance matrices of $\mathrm{ZF}$, such as capacity and BER, also has a limit, as the instantaneous SNR does.

\section{B. Simulation result}

We assume two co-located users and a BS with two antennas $(M=N=2)$, and all relays are co-located in midway between the source and BS. The results for both the fixed and variable gain schemes are shown in Fig. 2.

In Fig. 2, we observe $K=20$ relays approximate the limit for the fixed gain scheme. We also see that the performance of variable gain scheme does not reach the limit of the fixed gain scheme. Intuitively, when the instantaneous channel gain is smaller, the variable gain used by the AF relay will be large, so the noise at the relay will be amplified more compared with fixed gain. However, in practice, it's better to use variable gain instead of fixed gain because of the peak power constraint of the power amplifier. Especially, this is the
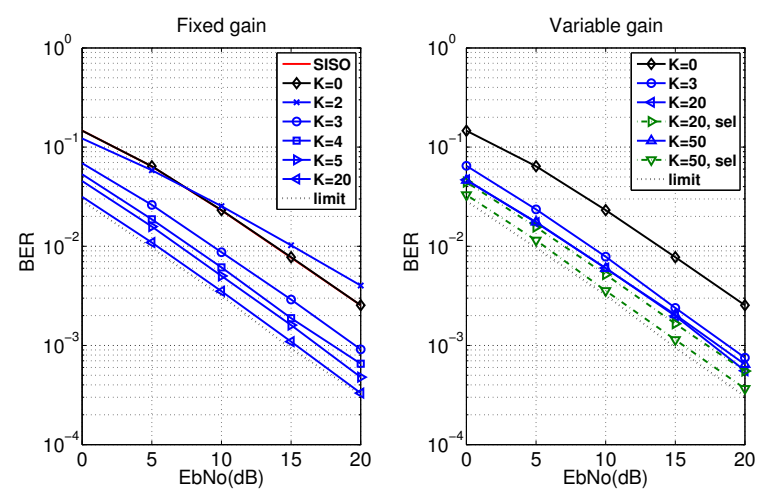

Figure 2: Monte Carlo simulation

case with OFDM, which is widely accepted to have large Peak-to-Average Power Ratio (PAPR).

One approach to improve the performance of the variable gain scheme is to use a selection strategy. Only the relays with gains less than a threshold are active. Here we choose the threshold to be the gain of the fixed gain scheme. The results for this selection strategy are shown in Fig.2 with legend "sel". We observe prominent improvement for the selection strategy with $K=50$.

\section{IMPLEMENTATION IN SOFTWARE-DEFINED RADIOS (SDRS)}

In our implementation, a wireless node consists of USRP2 [10], and GNU Radio [11] running on a personal computer (PC). The USRP2 has ADC/DAC and FPGA to digitize the analog waveform and create the baseband sampled data stream, which is sent to the PC through a gigabit ethernet cable. The PC does all the baseband signal processing based on the flowgraph we designed. All the blocks in the flowgraph are implemented in $\mathrm{C}++$ and integrated with Python scripts.

\section{A. Pre-synchronization}

In a distributed system, all the nodes within the same cluster or virtual antenna array should compensate their differences in both timing offset and carrier frequency offset (CFO). Therefore, before the source cluster starts sending data, we let the BS send a trigger signal $x_{t}$ directly to active users in source cluster, as a reference. All users then do pre-synchronization in both time and frequency domains based on this trigger signal. The BS could use transmit diversity to enable the trigger signal to reach the source cluster two hops away; instead, we just increase the transmit power of the trigger signal in our experiments.

We adopt a robust time pre-synchronization algorithm for cluster transmission, similar to that in [13]. All the 
cooperating nodes wait to transmit for a fixed period $T_{\text {proc }}$ after the end of the received trigger signal. So the transmiting time for $i$ th node within the cluster is

$$
T_{i}=T_{p}+t_{i}+T_{\text {proc }},
$$

where $T_{p}$ is the deterministic duration of trigger signal, $t_{i}$ represents the time the trigger waveform arrives at the antenna of the $i$ th node in the cluster; this is also called the "start of reception" (SOR), and the processing time $T_{\text {proc }}$ is selected so that $\alpha \times 100 \%$ of transmit firstin-first-out buffer will be full when the time comes to start the transmission. $\alpha$ is a design parameter such that $0 \leq \alpha \leq 1$. We use $\alpha=1$.

Another concern is the CFO caused by the difference of local oscillator. We propose that all nodes compensate the CFO to the data $x_{i}$ they plan to transmit by using numerically controlled oscillator (NCO) in SDRs. The transmit signal for $i$ th node becomes

$$
x_{i}^{\prime}(t)=x_{i}(t) e^{j 2 \pi \triangle f_{i} t},
$$

where $\triangle f_{i}$ is the estimated CFO of $i$ th node relative to $\mathrm{BS}$, which is negative of the detected CFO when the $i$ th node decodes the trigger signal $x_{t}$.

Nodes in the relay cluster schedule their transmissions using the same pre-synchronization method, but use the signal from source cluster as the trigger. Complete details about the distributed array OFDM pre-synchronization are given in [12].

\section{B. Source node}

We design each source to use OFDM modulation, with BPSK on each subcarrier. To implement the MUMIMO system with AF relays, we need several control parameters, such as the payload length, number of data streams in the network, cluster ID, and payload offset. However, the AF relays do not need channel state information (CSI) for the payload because it is not decoded. To deal with this conflict, we designed a physical (PHY) layer header, which is differentially encoded with control information and therefore can be decoded by AF relay without CSI; meanwhile, the header information can be read and updated during intermediate hops. Our packet consists of a preamble and a header; each using one OFDM symbol. This is followed by a training sequence of $M$ OFDM symbols, and finally a payload.

The $\mathrm{C}++$ blocks for generating a soource packet are shown in Fig. 3. When there are two parallel paths in the flowgraph, path 0 contains timing information, so that the source node knows the start of packet (SOP), and path 1 is the actual data stream. Otherwise, only data path

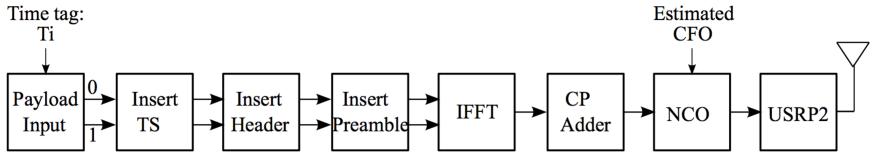

Figure 3: Flowgraph of source node

is processed by the block. A time tag with scheduled transmit time based on equation (11) is added at SOP in the first block. The source also sets estimated CFO parameter for the $\mathrm{NCO}$ block in the end of flowgraph to do frequency pre-synchronization when a packet is generated completely.

\section{AF relay transceiver}

The theory of AF is simple: amplify and re-transmit the received signal. However, from implementation point of view, AF requires some decoding. It needs some control information like packet length to coordinate the transmission. To solve this practical issue, we design the AF relay transceiver with receiver path including a DF branch and an AF branch, as shown in Fig. 4.

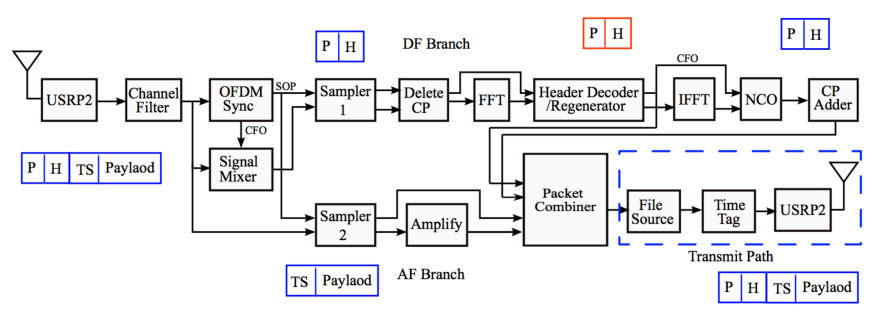

Figure 4: Flowgraph of AF relay transceiver

When a packet reaches the antenna of a relay, the OFDM Sync block does time and frequency recovery, and outputs SOP and CFO information. The input of the DF branch is the entire packet with CFO compensated using Signal Mixer block, while the input of AF branch is same but without CFO compensated. The Sampler 1 block selects out only the preamble and header data, while the Sampler 2 block selects out the TS and payload parts. In Header Decoder/Regenerator block of the DF branch, we decode the header to get all the control information, and then regenerate a new header with updated control information. Similarly as the presynchronization for source cluster, we use the $\mathrm{NCO}$ block to compensate the CFO in the newly generated header and preamble to keep the offsets consistent within a packet. The Packet Combiner block combines the new preamble and header data from DF branch and amplified payload from AF branch, and then stores them in file, which also works as the input of the transmit path of $\mathrm{AF}$ 
relay. Before the AF relay starts its transmit path, a time tag with scheduled transmit time information is added at the SOP for pre-synchronization in the time domain.

\section{MIMO receiver $(B S)$}

To build the MIMO receiver in SDRs, we synchronize multiple USRP2s using a reference clock distributor (RCD) board that we created in our lab. It provides 1 pulse per second (pps) timing reference and $10 \mathrm{MHz}$ frequency reference to USRP2s, so that all USRPs used at the receiver will have same time and frequency offset. The synchronized USRP2s are connected to a single PC to set up the MIMO receiver, as shown in Fig. 7c.

We implement a symmetric MU-MIMO system with the antennas of MIMO receiver equal to the number of users $(M=N)$. The ZF detection algorithm of VBLAST [14] is adopted as decoding algorithm for our MIMO receiver.

The receiver is implemented with the flowgraph shown in Fig. 5. For OFDM, the received signal is first synchronized by finding the SOP time and CFO, then the cyclic prefix (CP) is removed. After transforming the time domain signal back to the frequency domain by doing FFT, the OFDM Frame Acquisition block starts estimating the compound channel $\mathcal{H}$ directly based on TS, which go through the exactly same compound channel as payload. We implement the $\mathrm{ZF}$ algorithm in the OFDM Block Decoder block. The OFDM Frame Sink block demodulates the data using minimum distance detection, packs the decoded data, and then sends it to the higher layer.

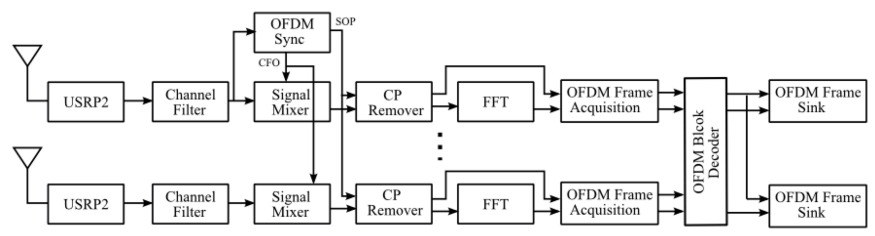

Figure 5: Flowgraph of MIMO receiver

\section{EXPERIMENT SETUP AND RESULTS}

\section{A. Experiment design}

We aim to show the performance improvement of MUMIMO with AF relays, in SDRs, by observing the BER or PER of MU-MIMO with different numbers of relays. The nodes are deployed (see Fig. 1) with 2 users, a relay cluster (up to 6 relay nodes), and MIMO receiver with 2 antennas, in a typical academic office building as shown in Fig. 6. We put the relay cluster symmetrically in the middle between the sources and the receiver. When number of relays $K \neq 0$, we let the relay node receive and re-transmit using a different carrier frequency to force the signal received by the BS go through relay cluster. The system parameters we use are shown in Table 1.

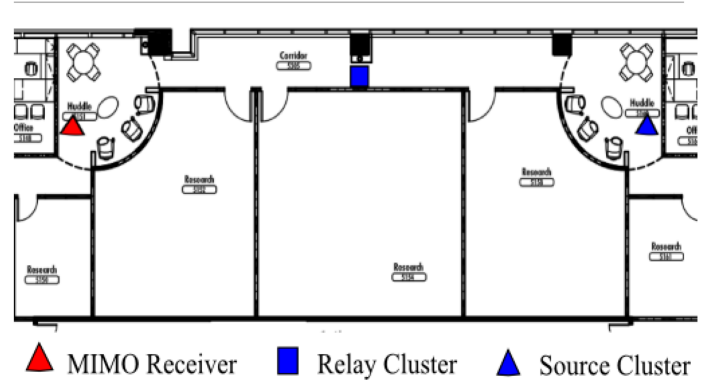

Figure 6: The floor plan with nodes deployment

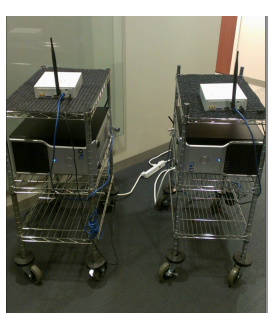

(a) Source nodes

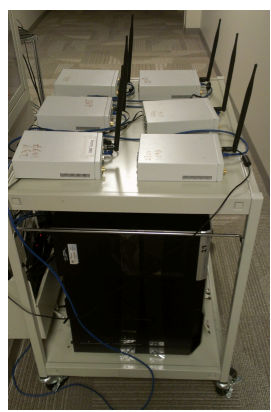

(b) Relay cluster

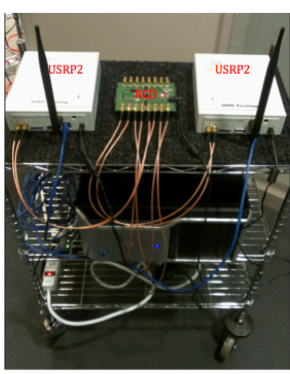

(c) MIMO receiver
Figure 7: Equipment setting

Table I: System parameters

\begin{tabular}{|c|c|}
\hline Modulation & BPSK \\
\hline Sampling rate & $1 \mathrm{MHz}$ \\
\hline FFT length & 104 \\
\hline Occupied subcarriers & 96 \\
\hline CP length & 8 \\
\hline
\end{tabular}

\section{B. Error rate measurement}

First, we compare the BER performance of MUMIMO without relays $(K=0)$ and with 2 AF relays $(K=2)$ in fixed locations, for different transmit powers $P_{0}$ and without error correction coding (ECC). In practical radio systems, it is easier to measure packet error rate (PER) rather than BER. The CRC error check determines if the received packet is corrupted. The estimated BER (EBER) can be calculated by EBER = $1-(1-P E R)^{1 / D}$ when no ECC is used, where $\mathrm{D}$ is the number of bits in a packet. For each transmit power, we measure PER averaged over about 2000 packets with 952 bits for every packet. The EBER curves (not shown 
because of space limitations) for transmit power ranging from $-10 \mathrm{dBm}$ to $-5 \mathrm{dBm}$, show an approximate straightline decrease on a semi-log graph, with the $K=0$ and $K=2$ cases nearly overlaying, except the $K=2$ case performs slightly better at $-10 \mathrm{dBm}$. For higher power or shorter range, the advantage of the relay gains is apparently outweighed by their inserted noise for $\mathrm{K}=2$.

To verify range extension, we test the PER at the receiver. During a 5 minute period, the receiver sends a trigger signal every $0.5 \mathrm{~s}$, and the number of relays increases every minute, from 2 to 6 . We look at transmit powers $P_{0}=-10 \mathrm{dBm},-8 \mathrm{dBm}$, and $-6 \mathrm{dBm}$, indicating bad, fair, and good EbNo at the receiver, and the PER is averaged over 10 trials.

In practice, self-fading is caused by relay timing error when multiple relays use the same channel resource. For the $2 \times 2$ case, we overcome this problem with half-rate convolutional ECC and interleaving. The PER results are shown in Fig. 8.
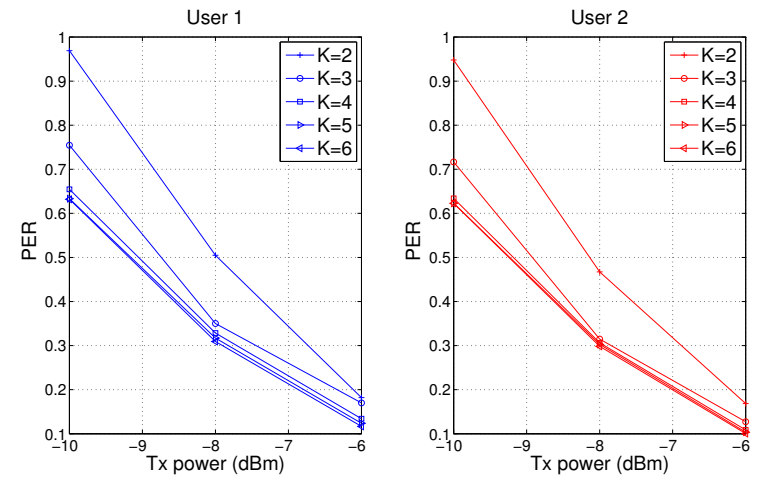

Figure 8: Averaged PER for MU-MIMO with different numbers of relays

Fig. 8 shows, the biggest improvement comes when we increase the number of relays from two to three, and improvements for more relays are small, which is similar to the simulation result with variable channel gain in Fig. 2. We observe that the experimental SNR improvement of only $1 \mathrm{~dB}$ is smaller than in the simulation; we think this may have been caused by interference that we noticed in this band after the experiment. We also observe that the slope is higher experimentally than in simulation. We attribute this to the frequency diversity in the experiment resulting from self and real fading and the ECC plus interleaving.

\section{CONCLUSION}

In this paper, we implement MU-MIMO with singleantenna AF relays in SDRs by specifically designing the AF tranceiver to overcome practical issues, such as presynchronization for cluster transmission. The averaged error rate is evaluated by both simulation and experiments with good correspondence. We prove theoretically that the effect of the growth of the number of fixed-gain relays is to bring the destination to the location of the relays. We find that applying a threshold test to variable gain relays (albeit more of them) yields nearly the same result as the fixed gain case.

\section{ACKNOWLEDGMENT}

This work was supported in part by Grant CNS1017248 from the National Science Foundation.

\section{REFERENCES}

[1] Qinghua Li; et al., "MIMO techniques in WiMAX and LTE: a feature overview," Communications Magazine, IEEE , vol.48, no.5, pp.86,92, May 2010.

[2] Miao Zhao; et al., "Mobile Data Gathering with Multiuser MIMO Technique in Wireless Sensor Networks," Global Telecommunications Conference, 2007. GLOBECOM '07. IEEE , vol., no., pp.838,842, 26-30 Nov. 2007.

[3] Chan-Byoung Chae; et al., "MIMO Relaying With Linear Processing for Multiuser Transmission in Fixed Relay Networks," Signal Processing, IEEE Transactions on , vol.56, no.2, pp.727,738, Feb. 2008.

[4] An Liu; et al., "Duality and Optimization for Generalized MultiHop MIMO Amplify-and-Forward Relay Networks With Linear Constraints," Signal Processing, IEEE Transactions on , vol.61, no.9, pp.2356,2365, May1, 2013.

[5] Berger; et al., "Cooperative distributed multiuser MMSE relaying in wireless ad-hoc networks." IEEE 39th Asilomar Conf. 2005.

[6] Wagner, J.; Wittneben, A., "On Capacity Scaling of MultiAntenna Multi-Hop Networks: The Significance of the Relaying Strategy in the Long Network Limit," Information Theory, IEEE Transactions on , vol.58, no.4, pp.2107,2133, April 2012

[7] Mavrokefalidis, C.; et al., "Optimal training design for channel estimation in OFDM/OQAM cooperative systems," Signal Processing Advances in Wireless Communications (SPAWC), 2013 IEEE 14th Workshop on , vol., no., pp.230,234, 16-19 June 2013.

[8] Rankov, et al.. "Distributed spatial multiplexing in a wireless network." Signals, Systems and Computers, 2004. Conference Record of the Thirty-Eighth Asilomar Conference on. Vol. 2. IEEE, 2004.

[9] John Proakis, Masoud Salehi, Digital Communications, 5th Edition, McGraw-Hill.

[10] http://gnuradio.org/redmine/projects/gnuradio/wiki/USRP2.

[11] http://gnuradio.org/redmine/projects/gnuradio/wiki.

[12] Yong Jun Chang, et al., "Pre-Synchronization for Multi-hop Distributed MIMO-OFDM," submitted to IEEE ICC 2014.

[13] Yong Jun Chang;et al., "Cluster Transmission Time Synchronization for Cooperative Transmission Using Software-Defined Radio," Communications Workshops (ICC), 2010 IEEE International Conference on, vol., no., pp.1,5, 23-27.

[14] Wolniansky, P.W.; et al., "V-BLAST: an architecture for realizing very high data rates over the rich-scattering wireless channel," ISSSE 98, vol., no., pp.295-300, 29 Sep-2 Oct 1998. 